\title{
Pleurotus ostreatus inhibits colitis-related colon carcinogenesis in mice
}

\author{
ANDREJ JEDINAK ${ }^{1}$, SHAILESH DUDHGAONKAR ${ }^{1}$, JIAHUA JIANG ${ }^{1}$, \\ GEORGE SANDUSKY ${ }^{2}$ and DANIEL SLIVA ${ }^{1,3,4}$ \\ ${ }^{1}$ Cancer Research Laboratory, Methodist Research Institute; ${ }^{2}$ Department of Pathology and Laboratory Medicine, \\ Simon Cancer Center, ${ }^{3}$ Indiana University Simon Cancer Center, School of Medicine, \\ ${ }^{4}$ Department of Medicine, Simon Cancer Center, Indiana University, Indianapolis, IN 46202, USA
}

Received June 14, 2010; Accepted July 20, 2010

DOI: 10.3892/ijmm_00000509

\begin{abstract}
Colorectal cancer is one of the leading causes of cancer deaths in both men and women in the world. However, colon cancer can be prevented to some extent by consumption of edible natural products with chemopreventive properties. Therefore, we investigated, whether edible mushroom Pleurotus ostreatus (PO) has chemopreventive effect on inflammation-associated colon carcinogenesis induced by 2-amino-1-methyl-6-phenylimidazo[4,5b]pyridine (PhIP) and promoted by dextran sodium sulfate (DSS). PO treatment, at both doses (100 and $500 \mathrm{mg} / \mathrm{kg}$ ), significantly reduced by 50 and $78 \%$ the number of aberrant crypt foci and the multiplicity of colon neoplasms by 43 and $89 \%$, respectively. However, incidence of colon tumors and high grade dysplasia was reduced by 50 and $63 \%$ only in the dose $500 \mathrm{mg} / \mathrm{kg}$ of $\mathrm{PO}$, respectively. Colon shortening and dysplastic index was significantly reduced by PO treatment in dose-dependent manner. The immunohistochemistry of colons revealed that treatment with $\mathrm{PO}$ suppressed expression of cyclin D1, Ki-67, COX-2 and F4/80. In summary, our data suggest that PO may prevent inflammation-associated colon carcinogenesis with exposure to PhIP through combined modulatory mechanisms of inflammation and tumor growth via suppression of COX-2, F4/80, Ki-67 and cyclin D1 expression in mice.
\end{abstract}

\section{Introduction}

Colorectal cancer (CRC) is one of the major public health problems in the United States and many other parts of the world. It is estimated that 106100 people in USA will be diagnosed with colon cancer and 49920 deaths will be

Correspondence to: Dr Daniel Sliva, Cancer Research Laboratory, Methodist Research Institute, 1800 N Capitol Avenue, E504, Indianapolis, IN 46202, USA

E-mail: dsliva@clarian.org

Key words: colorectal cancer, PhIP, Pleurotus ostreatus attributed to this disease in 2009 (1). CRC arises from a precursor lesion, the adenomatous polyp, which forms in a field of epithelial cell hyperproliferation and crypt dysplasia. Progression from this precursor lesion to CRC is a multistep process, accompanied by alterations in several suppressor genes that result in abnormalities of cell regulation that spans of 10-15 years (2). Recent data have showed that inflammation is a critical component of tumor progression. Many cancers arise from sites of infection, chronic irritation and inflammation. It is now becoming clear that the tumor microenvironment, which is largely orchestrated by inflammatory cells, is an indispensable participant in the neoplastic process, fostering proliferation, survival and migration $(3,4)$. Therefore, patients with inflammatory bowel disease (IBD)-either Crohn's disease (CD) or ulcerative colitis (UC)-are at increased risk for developing cancers of the gastrointestinal tract, particularly CRC (5). Of the environmental factors linked with human carcinogenesis, diet is regarded as a major determinant, in particular the consumption of meat (6). During the cooking of meat, mutagenic and carcinogenic heterocyclic amines are formed, the most abundant of which, 2-amino-1-methyl-6phenylimidazo[4,5-b]pyridine (PhIP), induces tumors of the prostate, colon and mammary gland in rats (7). The finding that PhIP induces cancer in colon tissue sites where tumors occur with high incidence in the Western world and the presence of PhIP in a Western-style diet rich in cooked meat (hamburgers, steaks, fried chicken or fish) suggest that this compound may pose a significant threat to human health. In support of this, PhIP-DNA adducts have been detected in human colon tissue (8). In addition, numbers of epidemiology studies have reported associations between consumption of red meat, PhIP intake, and cancer of the colon (9). Thus, humans are exposed to PhIP frequently through diet and lifestyle, and daily intake of PhIP among Americans has been estimated to be around 280-460 ng/d per person (10). Despite well developed diagnostic and therapeutic techniques, and novel anticancer drugs against CRC have been introduced, mortality rates of CRC have not remarkably been improved (11). Therefore chemoprevention strategies still appear as a possible alternative to screening and surveillance programs in reducing the incidence and the mortality for gastrointestinal cancer, including CRC at an acceptable cost/effectiveness ratio (12). This kind of strategy is gaining more attention. In 
particular, fungi can be used not only as strong agents with immunotherapeutic effects, but also as a source of potent metabolites, capable of penetrating cell membranes and interfering with particular signal transduction pathways linked to processes such as inflammation, cell differentiation and survival, carcinogenesis and metastasis (13).

Pleurotus ostreatus, oyster mushroom is an edible mushroom widespread throughout the hardwood forests of the world. A number of reports have demonstrated that $P$. ostreatus possess anticancer (14), antioxidant (15), hypocholesterolemic and antiatherogenic effects (16). The biologically active compounds responsible for anticancer and antioxidant effect isolated from $P$. ostreatus are polysaccharides, mainly $B-1,3-D$ glucans (17). Moreover, these polysaccharides have been linked to immunostimulatory (18) and antiinflammatory activity of $P$. ostreatus (19). Therefore, in our study, we examinated the chemopreventive effect of $P$. ostreatus against inflammation-associated colon carcinogenesis in mouse model utilizing a food-borne carcinogen PhIP and colitis-inducing agent dextran sulfate sodium salt (DSS) with respect to colon length, tumor incidence, multiplicity, dysplasia, neoplasia and aberrant crypt foci (ACF) formation. In addition, immunohistochemical expression of biomarkers involved in cancer progression (cyclin D1 and $\mathrm{Ki}-67)$ and biomarkers involved in modulation of inflammation (COX-2 and F4/80) were also evaluated in colonic tissues.

\section{Materials and methods}

Materials. Oyster mushroom (Pleurotus ostreatus) was provided by Forest mushrooms, Inc. (Saint Joseph, MN, USA). PhIP was purchased from Toronto Research Chemicals, Inc. (Toronto, Ontario, Canada) and dextran sulfate sodium salt (DSS) was obtained from MP Biomedicals (Solon, $\mathrm{OH}$, USA).

Preparation of oyster extract. The mushrooms ( $P$. ostreatus) were ground, then incubated for $24 \mathrm{~h}$ with absolute alcohol and the suspension was filtered on Whatman no. 3 paper to remove the biomass. This procedure was repeated twice. The supernatant was concentrated in a rotary evaporator at $50^{\circ} \mathrm{C}$ under reduced pressure as published before (14) and than stored at $4^{\circ} \mathrm{C}$.

Detection of lipopolysaccharides (LPS). Oyster mushroom extract was tested for LPS levels (Limulus Ameobocyte Lysate assay, Lonza, Walkersville, MD, USA). There was a low level of LPS contamination in the extract $(1.1 \mathrm{ng} / \mathrm{ml}$ in oyster extract). This concentration of LPS was well below the level of LPS required to stimulate cells in vitro (20).

Animals, chemicals and diets. Male Crj: CD-1 (ICR) mice (Harlan, Indianapolis, IN, USA) aged 5 weeks were used. They were maintained at Methodist Research Institute Animal Facility according to the Institutional Animal Care Guidelines. All animals were housed in plastic cages (4 mice/cage) with free access to drinking water and a pellet diet (LabDiet 5001, Southern Agriculture, Tulsa, OK, USA), under controlled conditions of humidity $(50 \pm 10 \%)$, light $(12 / 12 \mathrm{~h}$ light/dark

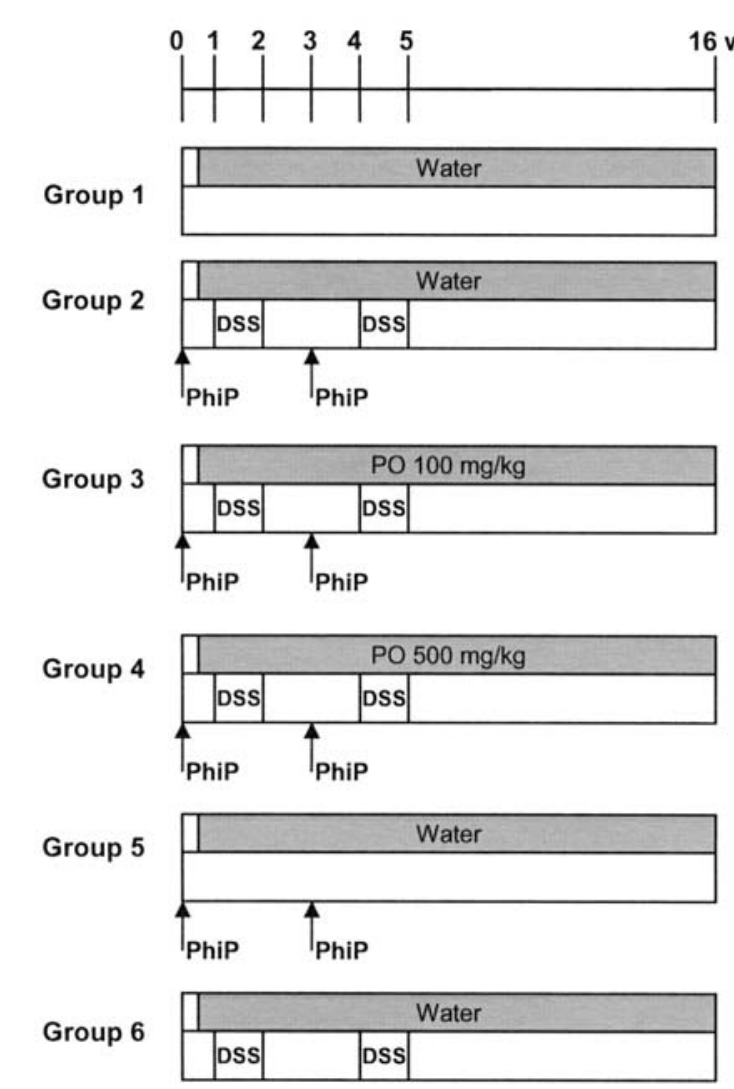

Figure 1. Experimental design.

cycle) and temperature $\left(23 \pm 2^{\circ} \mathrm{C}\right)$. After 7 days quarantine, they were randomized by body weight into experimental and control groups.

Experimental design. A total of 60 male ICR mice were divided into six groups (Fig. 1). Group $1(n=10)$ served as untreated control. The mice in groups $2(n=9)$ through 5 were initiated by a single itragastric gavage of PhIP $(200 \mathrm{mg} / \mathrm{kg}$ body weight). Starting 1 week after the injection, 2\% DSS in drinking water was administered to mice in groups 2 through 4 for 7 days. This procedure was repeated again 4 weeks later. In addition, groups $3(n=7)$ and $4(n=8)$ were gavaged three times a week (started next day after initiation of carcinogenesis by $\mathrm{PhIP}$ ) with 100 and $500 \mathrm{mg} / \mathrm{kg}$ of PO for 16 weeks, respectively. Group $5(\mathrm{n}=8)$ was given $\mathrm{PhIP}$ alone and group $6(n=9)$ received only $2 \%$ DSS in drinking water alone. All animals were euthanized at week 16 by $\mathrm{CO}_{2}$ asphyxiation and subjected to a complete gross necropsy examination to determine the incidence and multiplicity of tumors in large bowel. After measuring the colon length (from the ileocecal junction to the anal verge), colon was removed, rinsed with phosphatebuffered saline (PBS), and opened longitudinally. Three segments of $1.5 \mathrm{~cm}$ were taken from the proximal and distal part of the colon, fixed in $10 \%$ buffered formalin and embedded in paraffin. Sections $(4 \mu \mathrm{m})$ were cut and stained with hematoxylin-eosin. On H\&E stained sections, histological alterations, such as dysplasia and colonic neoplasms, were examined.

Scoring of aberrant crypt foci (ACF). For the counting of ACF unstained slides with paraffin-embedded tissues were 
selected as described before (17). Total three slides corresponding to three different animals (one slide per animal) from each group were used. The slides were then deparaffinized in xylene and rehydrated through graded alcohols to water. After removal of paraffin, tissues were stained with $0.2 \%$ methylene blue for $3 \mathrm{~min}$ in distilled water, and viewed under a light microscope to determine ACF. The ACF were distinguished from surrounding non-aberrant crypts by their increased size, thickened epithelial cell lining, and enlarged pericryptal area relative to surrounding normal crypts. Ten different foci (fields) were selected from each slide to count the aberrant crypts. The ACF from all 10 foci were added together for each slide. Lastly, average number of aberrant crypts from all 30 foci was determined together and percent response was calculated.

Histologic dysplasia scoring. Dysplasia was calculated by evaluating the severity of colon tissue damage as described before (21). The diagnostic categories represented were dysplasia not present (score 0), low-grade dysplasia (score 2) and high-grade dysplasia (score 4). After complete scoring the average score was determined as dysplastic index (DI).

Neoplastic index. Neoplastic index (NI) was calculated by evaluating the severity of neoplastic lesions like adenoma and or adenocarcinoma as described before (22). The actual index was calculated by scoring the neoplastic lesions in following manner. Mild/poorly developed, 1; moderately developed, 2; high/well developed, 3; very high/well developed, 4. After complete scoring the average score was determined as neoplastic index.

Immunohistochemistry. Immunohistochemistry for COX-2, F4/80, cyclin D1 and Ki-67 (Santa Cruz Biotechnology Inc., Santa Cruz, CA, USA) was performed on 3-mm thick paraffin-embedded sections from the colons of mice. For each case, negative controls were performed on serial sections. Antigen retrieval was performed by immersing the slides in Target Retrieval Solution (Dako) for $20 \mathrm{~min}$ at $90^{\circ} \mathrm{C}$ (in a water bath), cooling at room temperature for $10 \mathrm{~min}$, washing in water and then proceeding with immunostaining. All subsequent staining steps were performed by hand using Dako Reagents; incubations were done at room temperature and Tris-buffered saline plus 0.05\% Tween-20, pH 7.4 (TBS, Dako LSAB, Hamburg, Germany) was used for all washes and diluents. Thorough washing was performed after each incubation. Slides were blocked with protein blocking solution (Dako LSAB) for $25 \mathrm{~min}$; after washing, $10 \mu \mathrm{g} / \mathrm{ml}$ of the primary antibody was added to the slides and incubated for 60 min. A biotinylated link antibody plus streptavidinhorseradish peroxidase kit (Dako LSAB) was then utilized along with a DAB chromagen and peroxide substrate to detect the bound antibody complexes. The slides were briefly counterstained with hematoxylin, removed from the autostainer and dehydrated through graded alcohols to xylene. The slides were coverslipped with a permanent mounting media. Intensity and localization of immunoreactivities against all primary antibodies used were examined on all sections using a microscope (Leica DMR type 020-525-024 fluorescence microscope, Wetzlar, Germany). Total three slides

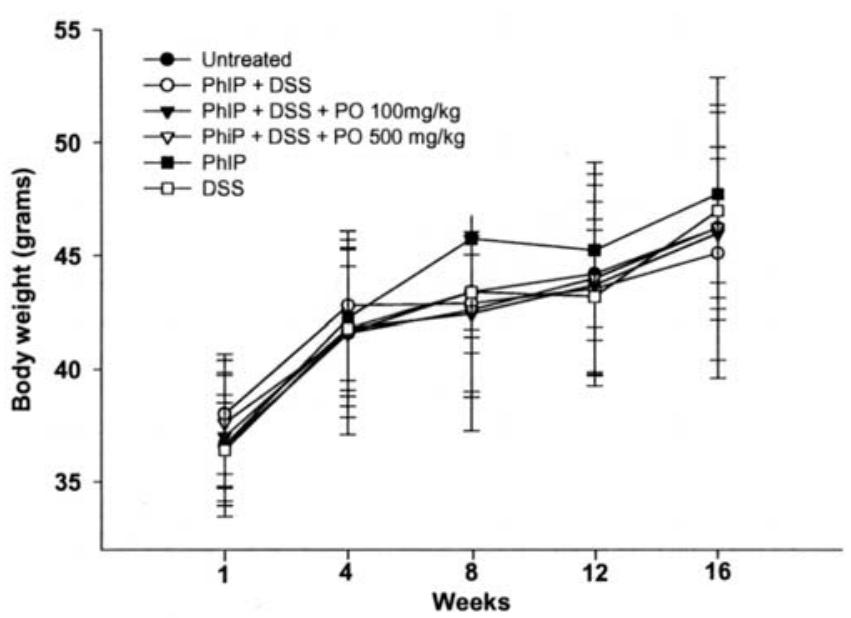

Figure 2. Body weight of mice. ICR mice were gavaged by PO with doses 100 or $500 \mathrm{mg} / \mathrm{kg}$ three times per week and body weight was taken every four weeks. Values are expressed as the means \pm SD

corresponding to three different animals (one slide per animal) from each group were used. Ten different foci (fields) were selected from the tumor periphery or untreated colonic tissue from each slide to count positive immunostained cells. All 10 foci were added together for each slide. Lastly, average number of positive cells from all 30 foci was determined together and percent response was calculated and recorded as published before.

Statistical analysis. Data were analyzed by Student's t-test. Fisher's Exact Probability test was used for comparison of the incidence of lesions between the two groups. Levels of cyclin D1, Ki-67, COX-2 and F4/80 in colon tissues were analyzed by ANOVA.

\section{Results}

General observation. The weight gain percentage over the entire study period is shown in Fig. 2. No significant differences were found in body weight between untreated group and the other groups. The weight gain percentage of mice in group $3(\mathrm{PhIP}+\mathrm{DSS}+\mathrm{PO} 100 \mathrm{mg} / \mathrm{kg})$ and group $4(\mathrm{PhIP}+$ DSS + PO $500 \mathrm{mg} / \mathrm{kg}$ ), which received P. ostreatus treatment were slightly higher than in the group $2(\mathrm{PhIP}+\mathrm{DSS})$, but not significant. Furthermore, the weight gain percentage of mice in group 5 (PhIP) and group 6 (DSS) were slightly higher than in group $2(\mathrm{PhIP}+\mathrm{DSS})$ as well. However, there was no change in liver and spleen weight and no hepatotoxic effect among those groups at the end of the study. During the study, diarrhea was found during and soon after DSS exposure (diarrhea started at 5th day up to 7th day) in mice that received $2 \%$ DSS in the drinking water and their body weight gains were slightly decreased. However, thereafter no such clinical symptoms were observed.

Colon length and dysplasia scores. Mouse colon length shrinks with stress, inflammation, ulceration or neoplasia. Therefore, mouse colon lengths were measured upon euthanasia. Oral administration of $P$. ostreatus significantly ameliorated short- 
Table I. Effect of $P$. ostreatus treatment on colon length, low and high grade dysplasia and dysplastic index.

\begin{tabular}{|c|c|c|c|c|c|}
\hline Groups & Treatments & $\begin{array}{c}\text { Colon } \\
\text { length }(\mathrm{cm})\end{array}$ & $\begin{array}{c}\text { Low grade } \\
\text { dysplasia score }\end{array}$ & $\begin{array}{c}\text { High grade } \\
\text { dysplasia score }\end{array}$ & Dysplastic index \\
\hline 1 & Control & $14.14 \pm 1.19$ & 0 & 0 & 0 \\
\hline 2 & $\mathrm{PhIP}+\mathrm{DSS}$ & $11.20 \pm 2.04^{\mathrm{a}}$ & $2.0 \pm 0$ & $3.56 \pm 1.33$ & $5.56 \pm 1.33$ \\
\hline 3 & $\mathrm{PhIP}+\mathrm{DSS}+\mathrm{PO} 100 \mathrm{mg} / \mathrm{kg}$ & $14.21 \pm 0.93^{\mathrm{b}}$ & $2.0 \pm 0$ & $2.29 \pm 2.14$ & $4.29 \pm 2.14$ \\
\hline 4 & $\mathrm{PhIP}+\mathrm{DSS}+\mathrm{PO} 500 \mathrm{mg} / \mathrm{kg}$ & $15.18 \pm 1.24^{\mathrm{c}}$ & $2.0 \pm 0$ & $1.33 \pm 2.00^{\mathrm{d}}$ & $3.33 \pm 2.0^{\mathrm{e}}$ \\
\hline 5 & PhIP & $14.49 \pm 1.14$ & $0.8 \pm 1.0$ & 0 & $0.86 \pm 1.07$ \\
\hline 6 & DSS & $11.58 \pm 1.78^{a}$ & $0.4 \pm 0.8$ & 0 & $0.44 \pm 0.88$ \\
\hline
\end{tabular}

Significantly different from control group $1,{ }^{a} \mathrm{p}<0.005$. Significantly different from group $2\left({ }^{b} p<0.005,{ }^{c} p<0.005 ;{ }^{d} p<0.05,{ }^{e} p<0.05\right)$.
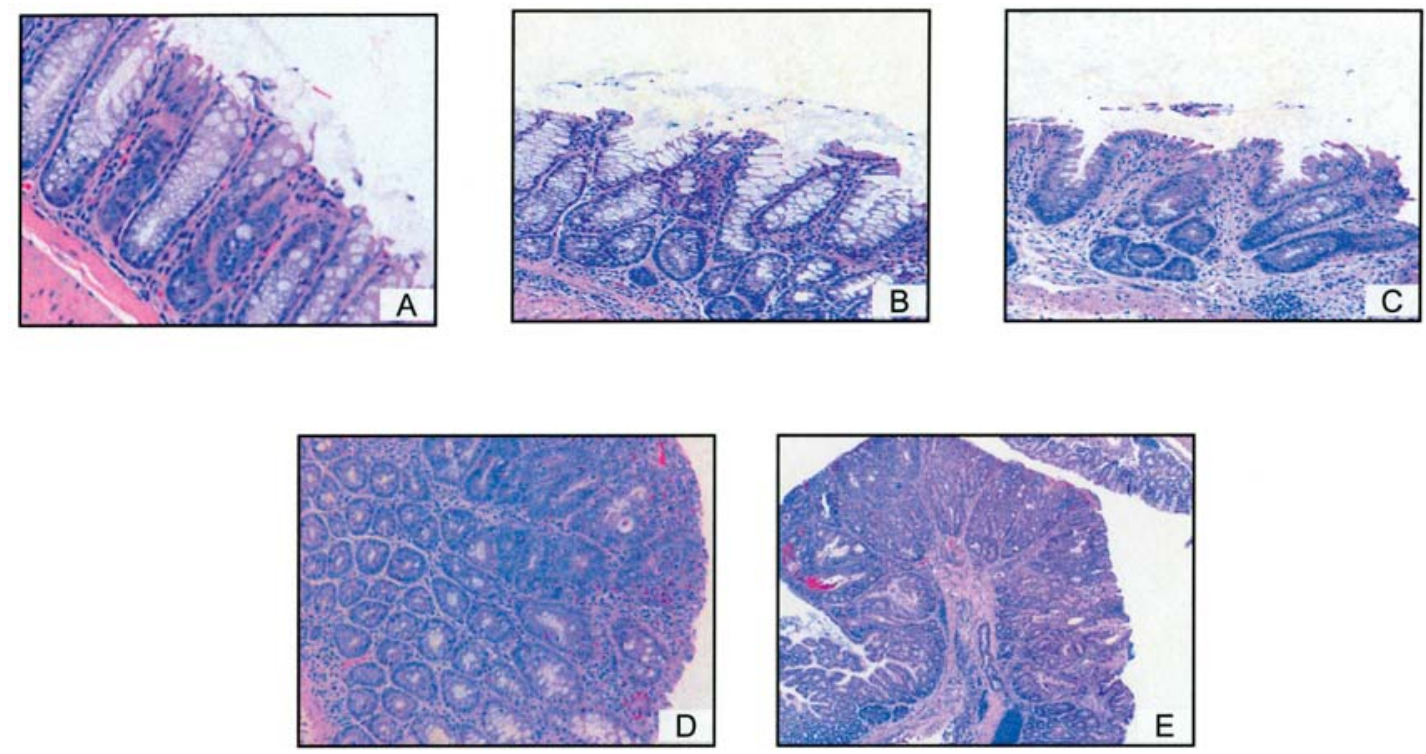

Figure 3. Histopathology of colonic lesions. (A) Normal colon tissue, (B) low-grade dysplasia, (C) high-grade dysplasia, (D) adenoma, (E) adenocarcinoma. H\&E stain, magnification x200 (A-C); magnification x100 (D-E).

enings of colon length compared with the positive control mice group $2(\mathrm{PhIP}+\mathrm{DSS})$ in dose-dependent manner (Table I). Results indicate that colon length of the large bowel of mice in the group 2 showed a markedly short colon length $(11.2 \pm 2.04 \mathrm{~cm}, \mathrm{p}<0.005)$ compared with the untreated group 1 $(14.14 \pm 1.19 \mathrm{~cm})$. The colon length in groups 3 and 4 , which received $P$. ostreatus treatment, was almost comparable to the untreated group 1. However, colon length in the groups 3 and 4 , which received $P$. ostreatus treatment was significantly longer (group 3: $14.21 \pm 0.93 \mathrm{~cm}, \mathrm{p}<0.005$; group 4: $15.18 \pm 1.24 \mathrm{~cm}, \mathrm{p}<0.005)$ than the colon length in the positive control group $2(\mathrm{PhIP}+\mathrm{DSS})$. Moreover, colon length in the group 6 (DSS) was significantly reduced $(11.57 \pm 1.77 \mathrm{~cm}$, $\mathrm{p}<0.005)$ compared to the untreated group $1(14.14 \pm 1.19$ $\mathrm{cm})$, but there was no effect of shortening in group 5 (PhIP alone) compared to group 1 . The colon shortenings were caused by DSS-induced inflammation in colonic mucosa. Gross examination of colon showed worse colon condition (colonic wall thickness, edema, hyperemia) in group 2 (PhIP + DSS) than in the groups 3 and 4 , which received $P$. ostreatus treatment. Histopathology (Fig. 3) revealed that there was no significant difference on low grade dysplasia between group 2 and groups which received $P$. ostreatus treatment, group 3 and group 4 (Table I). However, high grade dysplasia was significantly reduced by $63 \%(\mathrm{p}<0.05)$ in group $4(\mathrm{PhIP}+$ $\mathrm{DSS}+\mathrm{PO} 500 \mathrm{mg} / \mathrm{kg}$ ) compared to the positive control group 2 (PhIP + DSS). Moreover, high grade dysplasia in group 3 was reduced compared to group 2, but was not significant. In addition, dysplastic index was significantly reduced in group $4(\mathrm{p}<0.05)$ compared to group 2 .

Number of ACF and colonic neoplasms. Effect of P. ostreatus on aberrant crypt foci (ACF) formation, which are considered as precancerous lesions of the colon (23) is shown in Table II. The mice treated by PhIP + DSS (group 2) showed $100 \%$ $\mathrm{ACF}$ incidence, in contrast to the significant reduction in dosedependent manner in treatment group $3(\mathrm{p}<0.05)$ and group 4 $(\mathrm{p}<0.005)$. Moreover, nodular, polypoid or flat-type colonic tumors were observed in the middle and distal colon of all mice in groups 2 through 5 . There were no tumors in any 
Table II. Effect of $P$. ostreatus treatment on ACF, multiplicity of tumors, incidence of tumors and neoplastic index.

\begin{tabular}{llcccc}
\hline Groups & \multicolumn{1}{c}{ Treatments } & ACF & $\begin{array}{c}\text { Multiplicity } \\
\text { of tumors }\end{array}$ & $\begin{array}{c}\text { Incidence } \\
\text { (no. of mice with tumors) }\end{array}$ & $\begin{array}{c}\text { Neoplastic } \\
\text { index }\end{array}$ \\
\hline 1 & Control & 0 & 0 & $0 / 10(0)$ & 0 \\
2 & PhIP + DSS & $151.33 \pm 24.11$ & $5.56 \pm 2.01$ & $9 / 9(100)$ & $3.00 \pm 1.12$ \\
3 & PhIP + DSS + PO $100 \mathrm{mg} / \mathrm{kg}$ & $75.00 \pm 12.12^{\mathrm{a}}$ & $3.14 \pm 0.90^{\mathrm{c}}$ & $7 / 7(100)$ & $1.71 \pm 1.11^{\mathrm{f}}$ \\
4 & $\mathrm{PhIP}+\mathrm{DSS}+\mathrm{PO} 500 \mathrm{mg} / \mathrm{kg}$ & $33.33 \pm 8.39^{\mathrm{b}}$ & $0.63 \pm 0.74^{\mathrm{d}}$ & $4 / 8(50)^{\mathrm{e}}$ & $1.22 \pm 1.09 \mathrm{~g}$ \\
5 & $\mathrm{PhIP}$ & $23.00 \pm 12.49$ & $0.13 \pm 0.35$ & $1 / 8(13)$ & $0.57 \pm 0.53$ \\
6 & DSS & $22.00 \pm 9.85$ & 0 & $0 / 9(0)$ & $0.22 \pm 0.44$ \\
\hline
\end{tabular}

Significantly different from group 2 by Student's t-test. $\left({ }^{\mathrm{a}} \mathrm{p}<0.05,{ }^{\mathrm{b}} \mathrm{p}<0.005,{ }^{\mathrm{c}} \mathrm{p}<0.05,{ }^{\mathrm{d}} \mathrm{p}<0.005,{ }^{\mathrm{e}} \mathrm{p}<0.05,{ }^{\mathrm{f}} \mathrm{p}<0.05\right.$ and $\left.{ }^{\mathrm{g}} \mathrm{p}<0.005\right)$.
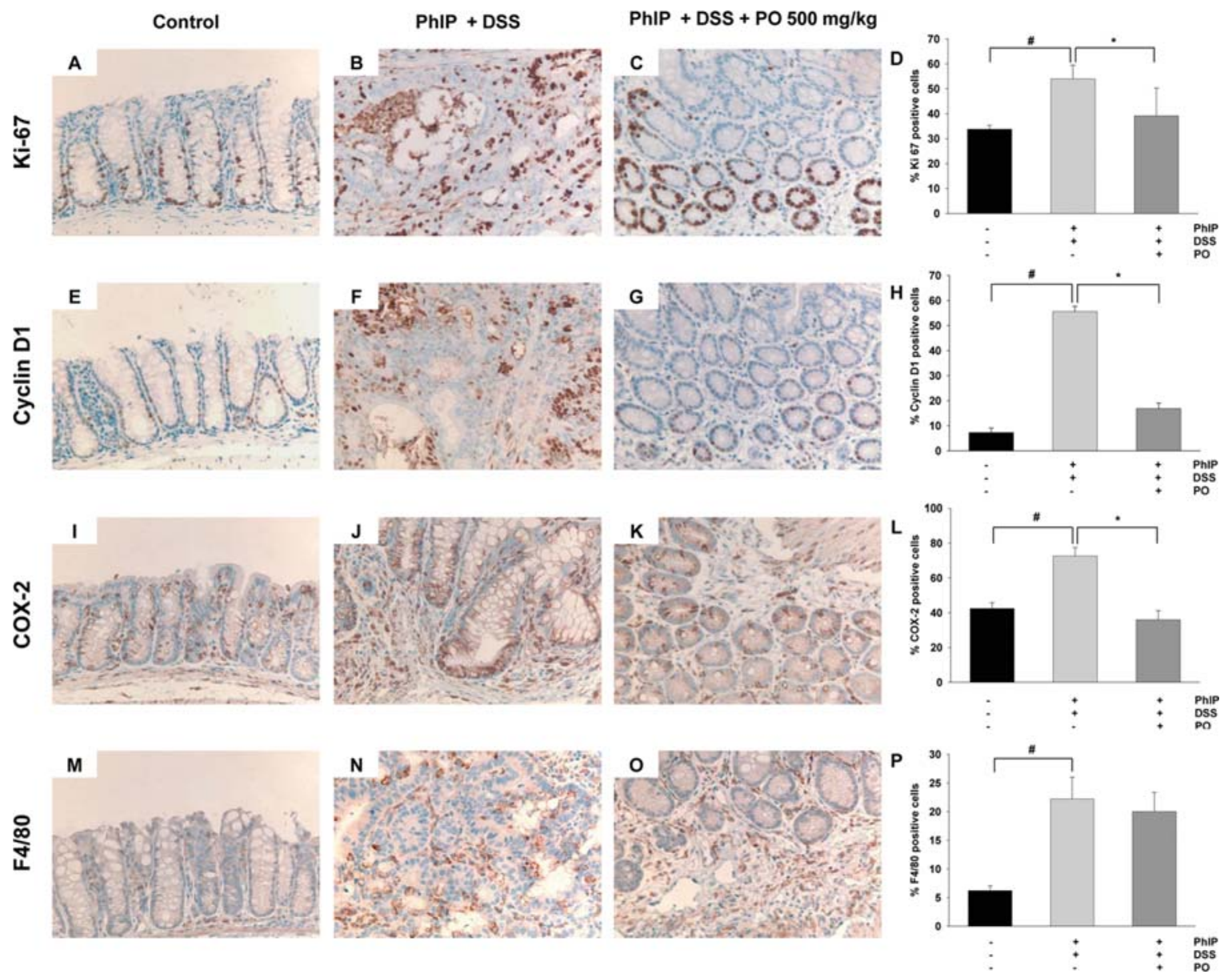

Figure 4. Immunohistochemistry and its quantification as percent of Ki-67, cyclin D1, COX-2 and F 4/80 positive cells in tumor periphery or in normal colonic tissue. Ki-67 immunohistochemistry (A-C) and its quantification (D), cyclin D1 immunohistochemistry (E-G) and its quantification (H), COX-2 immunohistochemistry (I-K) and its quantification (L), F 4/80 immunohistochemistry (M-O) and its quantification (P). Magnification used for all pictures was x200. Levels of cyclin D1, Ki-67, COX-2 and F4/80 in colon tissues were analyzed by ANOVA. "Statistically different from control group, p<0.05. *Statistically different from PhIP + DSS group, $\mathrm{p}<0.05$.

organs other than the large bowel. As shown in Table II, the multiplicity of tumors $5.56 \pm 2.01$ in group 2 was significantly reduced in a dose-dependent manner by $P$. ostreatus treatment to $3.14 \pm 0.9, \mathrm{p}<0.05$ and $0.63 \pm 0.74, \mathrm{p}<0.005$ in groups 3 and 4, respectively. As summarized in Table II, there was no difference in tumor incidence between group 2
(PhIP+DSS) and group 3 (PhIP + DSS + PO $100 \mathrm{mg} / \mathrm{kg})$. However, in group 4 the reduction was $50 \%(\mathrm{p}<0.05)$ of tumor incidence compared to group 2. Moreover, neoplastic index (NI) showed significant reduction in tumor progression in group $3(\mathrm{p}<0.05)$ and group $4(\mathrm{p}<0.005)$ compared to group 2. Furthermore, $P$. ostreatus administration markedly 
lessened the macroscopic and histological severity of the colonic wall. Moreover, the occurrences of those clinical signs were found to be delayed in P. ostreatus groups.

Immunohistochemistry for cyclin D1, Ki-67, COX-2 and F4/80 in colon tissues. To further investigate the impact of oyster mushroom we examined markers involved in cancer progression (Ki-67 and cyclin D1) and inflammatory markers (COX-2 and F4/80) by immunohistochemistry. Fig. 4 shows representative sections for all markers, which can be recognized by dark brown reaction with slight variation in intensity and distribution in tissues. Ki-67 is a well known marker for epithelial cell proliferation and was found elevated in the colon of female rats after PhIP treatment (24). Expression of Ki-67 was localized mainly to the nucleus. P. ostreatus treatment in group 4 significantly reduced Ki-67 expression, $\mathrm{p}<0.05$. Cyclin D1 is a cell cycle regulatory protein involved in cell cycle G1/S transition and was found elevated in rat mammary tumors initiated by PhIP (25). Immunohistochemical examination of cyclin D1 showed that abundance of this protein was markedly elevated in group $2(\mathrm{PhIP}+\mathrm{DSS})$ mainly in nuclei and partially in cytoplasma. However, in $P$. ostreatus treatment group 4 significant reduction was seen of cyclin D1 expression. Furthermore, we examined the expression of inflammatory markers COX-2 and F4/80. Cyclooxygenase-2 (COX-2) is a rate-limiting enzyme converting arachidonic acid to prostaglandins and often up-regulated in colorectal carcinogenesis (26). Moreover, COX-2 was found strongly expressed in tumors initiated by PhIP treatment (27). COX-2 immunoreactivity was strong mainly in the cell membrane and in the cell cytoplasm. Expression of COX-2 was significantly reduced by $P$. ostreatus in group 4 . F4/80 antigen is a $160-\mathrm{kDa}$ cell surface glycoprotein which expressed on a wide range of mature tissue macrophages (28). Strong immunostaining for F4/80 was localized mainly in the nucleus. Expression of F4/80 was reduced only slightly by $P$. ostreatus in group 4 and was not significant.

\section{Discussion}

We have previously described molecular mechanisms of $P$. ostreatus responsible for the inhibition of growth of breast and colon cancer cells through the modulation of cell cycle regulatory proteins via p53-dependent as well as p53independent pathway (14). Therefore, the goal of this pilot study was, whether $P$. ostreatus has the ability to reduce inflammation-associated colon carcinogenesis in vivo. This study represents the first findings that $P$. ostreatus inhibits food-borne carcinogen PhIP-induced colon carcinogenesis in a colitis-related mouse model. The findings described here clearly indicate the chemopreventive effect of P. ostreatus.

Patients with long-standing IBD have an increased risk of developing CRC (29). The histopathogenesis of IBD-associated colorectal carcinogenesis is widely believed to involve a step-wise progression from inflamed and hyperplastic cryptal cells through flat dysplasia to finally adenocarcinoma (30). Moreover, the neoplastic transformation in IBD is thought to be similar to the adenoma-carcinoma sequence in sporadic colon cancer (SCC). However, unlike SCC, where dysplastic lesions arise in one or two focal areas of the colon, in colitic mucosa, it is not unusual for dysplasia or cancer to be multifocal, reflecting a broader 'field effect' (29). Additionally, the molecular biology of cancer in IBD is unique in that the accumulation of molecular and genetic alterations may occur more rapidly or in an unconventional sequence when compared to sporadic CRC (31). Many of the molecular alterations responsible for sporadic colorectal cancer, namely chromosomal instability, microsatellite instability and hypermethylation, also play a role in colitis-associated colon carcinogenesis (29). Given the limitations of the current surveillance approach (mainly colonoscopy) or adverse effects of corticosteroids and non-steroidal anti-inflammatory drugs, primary cancer prevention via chemoprevention has been proposed as an alternative or additive strategy. Another advantage of chemoprevention over the current secondary prevention strategy of routine colonoscopy is the potential to intervene early enough in the carcinogenic sequence to avoid not only cancer, but also the need for colectomy. The goal of chemoprevention is to reduce CRC risk, allowing for less frequent surveillance exams and a reduction in the number of invasive cancers. Therefore, cancer risk reduction through regular use of chemopreventive medications remains an attractive concept (31).

During the entire study $P$. ostreatus did not cause any significant change in body weight of mice and there were no side effects in mice treated with oyster mushroom. The DSSinduced model of colitis is associated with loss of weight, diarrhea, blood in the stool and shortening of the colon (32). In addition, measurement of colon length is often used as a morphological parameter for the degree of inflammation in DSS induced colitis (33). Previously, it was reported that pleuran (ß-1,3-D glucan) from P. ostreatus reduced colitis in rats induced by intracolonic administration of $4 \%$ acetic acid $(19,34)$. However, effect of $P$. ostreatus on the colon length was not evaluated in those studies. Thus, we measured the colon length at the end of the study and we found that treatment with $P$. ostreatus prevented shortening of the colon as one of the parameters in colitis. Therefore, the ability of $P$. ostreatus to prevent shortening of the colon could suggest an anti-inflammatory effect of oyster mushroom. Much interest is currently directed toward research in the use of surrogate end-point biomarkers that are altered early in colonic carcinogenesis, before polyp (adenoma) formation, to predict the clinical effectiveness of chemopreventive agents or drugs because it takes 10-20 years for a normal cryptal cell to undergo molecular changes to be clinically detected as a neoplasm. Aberrant crypt foci (ACF) can be used as the endpoint in CRC development because aberrant crypts are postulated to be the earliest identifiable potential precursors of CRC in rodents and humans (11). Bobek and Galbavy (17) reported that treatment by pleuran ( $ß-1,3-\mathrm{D}$ glucan) from $P$. ostreatus in $\mathrm{DMH}$-induced colon carcinogenesis in rats reduced the incidence of ACF lesions in rat colon and increased superoxide dismutase (SOD) activity in liver, catalase (CAT) activity in erythrocytes and glutathione peroxidase (GSH-PX) in the colon due to it is antioxidative effect. Here we report that $P$. ostreatus treatment significantly reduced ACF formation in both treatment groups in a dose-dependent manner in our mouse model. In addition, chemopreventive agent $\alpha$-naphthyl isothiocyanate has been reported to increase the activity of 
GST which can inhibit DNA binding of N-acetyl-PhIP and thus suppress colon carcinogenesis (35). Therefore, the ability of pleuran ( $(1,1,3-\mathrm{D}$ glucan) from $P$. ostreatus to increase GST levels may also reduce metabolic activation of $\mathrm{PhIP}$ and thus reduce colon carcinogenesis as well. Another morphological parameter of neoplastic precursor lesions is dysplasia. The term dysplasia is used to describe structural and cytological alterations in the epithelium that predispose an organ to cancer development (11). Cyclic administration of DSS in drinking water results in the establishment of chronic colitis and the development of colorectal dysplasias and cancers with pathological features that resemble those of human colitis-associated neoplasia (36). Therefore, we evaluated effect of $P$. ostreatus on low-grade and high-grade dysplasia in colon of mice. Histopathology revealed that $P$. ostreatus treatment did not have significant effect on lowgrade dysplasia, but significantly reduced high grade dysplasia at $500 \mathrm{mg} / \mathrm{kg}$. Moreover, dysplastic index was also significantly reduced in the $500 \mathrm{mg} / \mathrm{kg}$ dose of $P$. ostreatus treatment. Colonic neoplasm-nodular, polypoid or flat-type colonic tumors were observed in the middle and distal colon in all groups except the control and DSS group. We found that $P$. ostreatus significantly reduced multiplicity and also incidence of colon neoplasm in the dose $500 \mathrm{mg} / \mathrm{kg}$ of oyster mushroom. Our results are in agreement with previous studies also demonstrating the reduction in tumor incidence by $P$. ostreatus in DMH induced CRC $(37,38)$. Our data suggest that $P$. ostreatus posses anti-inflammatory and anticancer properties as well. Therefore, to better understand anti-inflammatory and anti-cancer mechanism of $P$. ostreatus, we performed immunohistochemistry analysis of COX-2, F4/80, Ki-67 and cyclin D1 as biomarkers involved in inflammation and cancer of the colon. Immunohistochemistry revealed that treatment with $P$. ostreatus significantly decreased expression of COX-2 and slightly decreased macrophage infiltration by reducing expression of F4/80. In addition, $P$. ostreatus treatment significantly downregulated expression of biomarkers involved in colon cancer progression cyclin D1 and Ki-67. The results clearly indicate that administration of $P$. ostreatus prevented PhIP/DSSinduced mouse colitis-related colonic tumorigenesis without any adverse effects.

In summary, the administration of $P$. ostreatus effectively suppressed PhIP/DSS-induced colon carcinogenesis by reduction of COX-2, F4/80, Ki-67 and cyclin D1 expression in mice colon tissues. These results indicate that edible oyster mushroom might suppress IBD-associated colon carcinogenesis and is possibly applicable for human clinical trials.

\section{Acknowledgements}

This study was supported by the pilot grant to D.S. from the Purdue-UAB Botanicals Research Center for age-related diseases (P50 AT00477, Connie M. Weaver, PI) from the National Center for Complementary and Alternative Medicine and the NIH Office of Dietary Supplements.

\section{References}

1. Jemal A, Siegel R, Ward E, Hao Y, Xu J and Thun MJ: Cancer statistics, 2009. CA Cancer J Clin 59: 225-249, 2009.
2. Winawer SJ: Natural history of colorectal cancer. Am J Med 106: S3-S6, 1999.

3. Coussens LM and Werb Z: Inflammation and cancer. Nature 420: 860-867, 2002.

4. Jedinak A, Dudhgaonkar S and Sliva D: Activated macrophages induce metastatic behavior of colon cancer cells. Immunobiology 215: 242-249, 2010.

5. Lichtenstein GR: Reduction of colorectal cancer risk in patients with Crohn's disease. Rev Gastroenterol Disord 2: 16-24, 2002.

6. Food, Nutrition, Physical Activity and the Prevention of Cancer (WCRF): A Global Perspective. Washington (DC). Am Inst Cancer Res pp1-517, 2009.

7. Creton SK, Zhu H and Gooderham NJ: The cooked meat carcinogen 2-amino-1-methyl-6-phenylimidazo[4,5-b]pyridine activates the extracellular signal regulated kinase mitogenactivated protein kinase pathway. Cancer Res 67: 11455-11462, 2007.

8. Malfatti MA, Dingley KH, Nowell-Kadlubar S, Ubick EA, Mulakken N, Nelson D, Lang NP, Felton JS and Turteltaub KW: The urinary metabolite profile of the dietary carcinogen 2-amino1-methyl-6-phenylimidazo[4,5-b]pyridine is predictive of colon DNA adducts after a low-dose exposure in humans. Cancer Res 66: 10541-10547, 2006.

9. Sinha R, Peters U, Cross AJ, Kulldorff M, Weissfeld JL, Pinsky PF, Rothman N and Hayes RB: Meat, meat cooking methods and preservation, and risk for colorectal adenoma. Cancer Res 65: 8034-8041, 2005.

10. Byrne C, Sinha R, Platz EA, Giovannucci E, Colditz GA, Hunter DJ, Speizer FE and Willett WC: Predictors of dietary heterocyclic amine intake in three prospective cohorts. Cancer Epidemiol Biomarkers Prev 7: 523-529, 1998.

11. Tanaka T: Colorectal carcinogenesis: review of human and experimental animal studies. J Carcinog 8: 5, 2009.

12. Bazuro GE, Torino F, Gasparini G and Capurso L: Chemoprevention in gastrointestinal adenocarcinoma: for few but not for all? Minerva Gastroenterol Dietol 54: 429-444, 2008.

13. Petrova RD, Reznick AZ, Wasser SP, Denchev CM, Nevo E and Mahajna J: Fungal metabolites modulating NF-kappa B activity: an approach to cancer therapy and chemoprevention. Oncol Rep 19: 299-308, 2008

14. Jedinak A and Sliva D: Pleurotus ostreatus inhibits proliferation of human breast and colon cancer cells through p53-dependent as well as p53-independent pathway. Int J Oncol 33: 1307-1313, 2008.

15. Bobek P, Ozdin L and Kuniak L: Antioxidative effect of oyster mushroom (Pleurotus ostreatus) in hypercholesterolemic rat. Pharmazie 50: 441-442, 1995.

16. Bobek P and Galbavy S: Hypocholesterolemic and antiatherogenic effect of oyster mushroom (Pleurotus ostreatus) in rabbits. Nahrung 43: 339-342, 1999.

17. Bobek P and Galbavy S: Effect of pleuran (beta-glucan from Pleurotus ostreatus) on the antioxidant status of the organism and on dimethylhydrazine-induced precancerous lesions in rat colon. Br J Biomed Sci 58: 164-168, 2001.

18. Sun Y and Liu J: Purification, structure and immunobiological activity of a water-soluble polysaccharide from the fruiting body of Pleurotus ostreatus. Bioresour Technol 100: 983-986, 2009.

19. Nosálová V, Bobek P, Cerná S, Galbavy S and Stvrtina S: Effects of pleuran (beta-glucan isolated from Pleurotus ostreatus) on experimental colitis in rats. Physiol Res 50: 575-581, 2001.

20. Dudhgaonkar S, Thyagarajan A and Sliva D: Suppression of the inflammatory response by triterpenes isolated from the mushroom Ganoderma lucidum. Int Immunopharmacol 9: 1272-1280, 2009.

21. Xiao H, Hao X, Simi B, Ju J, Jiang H, Reddy BS and Yang CS: Green tea polyphenols inhibit colorectal aberrant crypt foci (ACF) formation and prevent oncogenic changes in dysplastic ACF in azoxymethane-treated F344 rats. Carcinogenesis 29: 113-119, 2008.

22. Nakagama H, Nakanishi $\mathbf{M}$ and Ochiai M: Modeling human colon cancer in rodents using a food-borne carcinogen, PhIP. Cancer Sci 96: 627-636, 2005.

23. Bird RP: Observation and quantification of aberrant crypts in the murine colon treated with a colon carcinogen: preliminary findings. Cancer Lett 37: 147-151, 1987.

24. Ikeda Y, Cho YM, Takahashi S, Tang M, Asamoto M, Ogawa K and Shirai T: Equivocal impact of transplacental and lactational exposure to a food-derived carcinogen, 2-amino-1-methyl-6phenylimidazo[4,5-b]pyridine, on prostate and colon lesion development in F344 rats. Cancer Lett 224: 23-30, 2005. 
25. Qiu C, Shan L, Yu M and Snyderwine EG: Deregulation of the cyclin $\mathrm{D} 1 / \mathrm{Cdk} 4$ retinoblastoma pathway in rat mammary gland carcinomas induced by the food-derived carcinogen 2-amino-1methyl-6-phenylimidazo[4,5-b]pyridine. Cancer Res 63: 5674-5678, 2003.

26. Doherty GA and Murray FE: Cyclooxygenase as a target for chemoprevention in colorectal cancer: lost cause or a concept coming of age? Expert Opin Ther Targets 13: 209-218, 2009.

27. Tanaka T, Suzuki R, Kohno H, Sugie S, Takahashi M and Wakabayashi K: Colonic adenocarcinomas rapidly induced by the combined treatment with 2-amino-1-methyl-6-phenylimidazo [4,5-b]pyridine and dextran sodium sulfate in male ICR mice possess beta-catenin gene mutations and increases immunoreactivity for beta-catenin, cyclooxygenase- 2 and inducible nitric oxide synthase. Carcinogenesis 26: 229-238, 2005.

28. Gordon S, Crocker PR, Morris L, Lee SH, Perry VH and Hume DA: Localization and function of tissue macrophages. Ciba Found Symp 118: 54-67, 1986.

29. Xie J and Itzkowitz SH: Cancer in inflammatory bowel disease. World J Gastroenterol 14: 378-389, 2008

30. Riddell RH, Goldman H, Ransohof DF, Appleman HD, Fenoglio CM, Haggitt RC, Ahren C, Correa P, Hamilton SR, Morson BC, Sommers SC and Yardley JH: Dysplasia in inflammatory bowel disease: standardized classification with provisional clinical application. Human Pathol 14: 931-968, 1983.

31. Zisman TL and Rubin DT: Colorectal cancer and dysplasia in inflammatory bowel disease. World J Gastroenterol 14: 2662-2669, 2008.
32. Hendrickson BA, Gokhale R and Cho JH: Clinical aspects and pathophysiology of inflammatory bowel disease. Clin Microbiol Rev 15: 79-94, 2002.

33. Kwon HS, Oh SM and Kim JK: Glabridin, a functional compound of liquorice, attenuates colonic inflammation in mice with dextran sulphate sodium-induced colitis. Clin Exp Immunol 151: 165-173, 2008.

34. Bobek P, Nosálová V and Cerná S: Effect of pleuran (betaglucan from Pleurotus ostreatus) in diet or drinking fluid on colitis in rats. Nahrung 45: 360-363, 2001.

35. Sugie S, Ohnishi M, Ushida J, Yamamoto T, Hara A, Koide A, Mori Y, Kohno H, Suzuki R, Tanaka T, Wakabayashi K and Mori H: Effect of alpha-naphthyl isothiocyanate on 2-amino-3methylimidazo[4,5-b]pyridine (PhIP)-induced mammary carcinogenesis in rats. Int J Cancer 115: 346-350, 2005.

36. Clapper ML, Cooper HS and Chang WC: Dextran sulfate sodium-induced colitis-associated neoplasia: a promising model for the development of chemopreventive interventions. Acta Pharmacol Sin 28: 1450-1459, 2007.

37. Zusman I, Reifen R, Livni O, Smirnoff P, Gurevich P, Sandler B, Nyska A, Gal R, Tendler Y and Madar Z: Role of apoptosis, proliferating cell nuclear antigen and p53 protein in chemically induced colon cancer in rats fed corncob fiber treated with the fungus Pleurotus ostreatus. Anticancer Res 17: 2105-2113, 1997.

38. Bobek P, Galbavy S and Ozdin L: Effect of oyster mushroom (Pleurotus ostreatus) on pathological changes in dimethylhydrazine-induced rat colon cancer. Oncol Rep 5: 727-730, 1998. 